family, with the additional problems at home arising from the excessive washing involved. It is no comfort to child or parent to be told, "He will grow out of it."

We feel, then, that it is unfair to dismiss a potentially useful treatment so sweepingly. Surely it would be more sensible for us to aim to educate parents to treat the drugs with caution, and to make more use of childproof containers both for syrup and for tablets.

SusAN D Dickson

Enuresis Clinic,

Doncaster Royal Infirmary,

P DICKSON

Hatfield,

Doncaster, Yorks DN7 6JX

SIR,-Against the growing sophistication of modern educational institutions the effects of persistent bed-wetting in terms of the emotional and social development of enuretic sufferers are not always "benign," as your leading article (17 March, p 706) states. May I therefore suggest an approach which can readily be used by clinical medical officers in enuresis clinics operating from school health premises ?

In cases of persistent enuresis cystometric examination of bladder function indicates that in the instances examined bed-wetting is the consequence of a developmental disorder of the bladder musculature. ${ }^{1}$ If therefore the tricyclic antidepressants imipramine and amitryptiline are used for their direct action on smooth muscle ${ }^{2}$ success rates of above $90 \%$ can be obtained. In this context the timing of administration is more important than the actual dosage; and by combining chemotherapy with bladder exercises, such as repeated bladder distension and voluntary retention of urine, the relapse rate can be reduced to acceptable proportions.

In general, the greater the age of the child the better the outlook, because in the older child his or her active co-operation can more readily be obtained.

GWYNNE V LEWIS

$$
\begin{aligned}
& \text { Health Department, } \\
& \text { Ipswich, Suffolk } \\
& \\
& { }^{1} \text { Bakwin, H, and Bakwin, R M, Behaviour Disorders in } \\
& \text { Children, p 442. London, W B Saunders Company, } \\
& 1972 . \text { S and De Quenedo, A, Disease. American } \\
& \text { Epstein, S J, and De } \\
& \text { fournal of Psychiatry, 1964, 120, 908. }
\end{aligned}
$$

\section{Malaria in Wolverhampton}

SIR,-We were very interested in the communications from Dr Eric Walker (16 December, $p$ 1718) and Dr C J Ellis and others (10 February, p 385) and would like to add our experience.

For the year ending 31 December 1978, we have diagnosed 158 new cases of malaria within the area of our health authority, compared with 112 cases in 1977 - a $42 \%$ increase, reflecting the present upsurge of this infection in the tropics. There have been no deaths from this infection. One hundred and twenty-six $(80 \%)$ cases presented between May and September. Seventy-six ( $48 \%$ ) cases were new immigrants. All but one case were from the Indian subcontinent and infected with Plasmodium vivax. The exception was a 30-year-old African male from Malawi who was infected with $P$ ovale. Three $(2 \%)$ Asians making a holiday visit to this country were also infected with $P$ vivax. The other $79(50 \%)$ cases were residents of this country who had been travelling abroad. One man who was infected with $P$ falciparum was a British worker returning from Nigeria. The remainder, most of whom were infected with $P$ vivax, were visitors to the Indian subcontinent, mainly the Punjab. However, there were two adults infected with $P$ falciparum (one of whom had a mixed infection with $P$ vivax) and three cases of $P$ malariae infection (one was mixed with $P$ vivax). Interestingly, about one-quarter $(19 / 78)$ of the travellers to the Indian subcontinent were children under 14 years of age. Those who were born in the UK may be more vulnerable to the dangers complicating infection with $P$ falciparum.

These figures of course represent only cases confirmed by examination of stained blood films in our laboratory and thus are baselines. It may be that there are many more cases which are diagnosed and treated clinically but which are not notified.

Our experience and that of others clearly demonstrates that the problem is increasing; it could be considerably reduced if much more attention were focused on informing travellers about this disease and its possible danger and educating them about its prevention. Publicity could be effectively promoted by campaigns carried out by the advertising media, with appropriate medical advice to ensure accuracy and relevance of information Such schemes, at least during peak travelling months, should include television advertisements, press articles, and talks on local radio stations, particularly if they are reinforced in the local dominant Asian language as well as English. General advice and prescriptions for prophylactic drugs are available from genera practitioners. However, prophylactic drugs are not available at the Health Service's expense, and so it should be publicised that they are cheap. Should these drugs be made available under prescribing regulations? It is most important to emphasise too that drugs must be taken continuously during the period abroad and for a period of at least a month after return to this country.

Such positive measures should bring to the overstretched resources of the NHS the additional reward of conserving valuable clinical time and services.

We thank our colleagues in the Malaria Reference Laboratory for their invaluable help.

Public Health Laboratory, ( Wolverhampton WV10 0QP

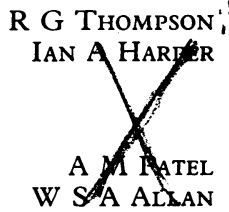

Haematology Laboratories,
Wolverhampton Area Health Authority

Liver injury, drugs, and popular poisons

SIR,-I was very interested to see the leading article on the wide range of drugs that affect the liver ( 3 March, p 574) but a little disappointed to read the following: "and after years of controversy halothane has now earned a definite place in the list of drugs causing liver disease." As an anaesthetist who greatly values halothane, I do not eliminate the possibility that it may (very rarely) adversely affect the liver, but I wish that hepatologists and others would be a little more open minded.

The implication of that statement is that there is a clearly proved case against the drug, and no longer any controversy. Reference to a paper ${ }^{1}$ by Sherlock is made in support of the statement. The paper in question, however, was included in the Lancet under the genera heading "Point of view." Surely one person's opinion, even an opinion as eminent as Pro- fessor Sherlock's, cannot be taken as the basis for such a dogmatic statement.

The article goes on to review the many proprietary preparations available that may affect the liver. One wonders how many of the patients labelled as having halothane hepatitis have had the possibility of ingestion of such agents eliminated from their histories.

\section{J A W WILDSMITH}

Department of Anaesthetics,

Royal Infirmary,

' Sherlock, S, Lancet, 1978, 2, 364

\section{Prescription for a better British diet}

SIR,-Dr $\mathbf{R}$ Passmore and others prescribe "for a better British diet" (24 February, p 527) and undoubtedly they succeed, but it is not the best. Though they base their prescription on present food supplies in the UK, they should have heeded the dietary goals prepared by the McGovern Committee for the US ${ }^{1}$ which are generally applicable to the UK.

Passmore et al make no recommendation about the use of high-extraction flour or of polyunsaturated fats. They state that bread "is rich in many nutrients"; but $87 \%$ of the bread being eaten is white, ${ }^{2}$ to which this statement hardly applies in comparison with bread made from high-extraction flour (which also contains fibre). The laudable reduction in meat will reduce saturated fat, but the overall reduction of $15 \%$ in total fat will not increase essential fatty acids (EFA, or certain polyunsaturated fatty acids). This increase is wisely recommended by McGovern. Dr J I Mann (17 March, p 732) follows McGovern in suggesting that the ratio of saturated to polyunsaturated fatty acids should be unity, but I join with Sir John McMichael (20 January, $p$ 173) against Mann in disliking transpolyunsaturated fatty acids (which are not EFA and tend to behave biologically as (saturated). Mann's statement that "diets rich in saturated fat are invariably high in refined carbohydrate and total energy intake" is not correct. Eskimos and mutton-eating Welsh in Patagonia had diets high in saturated fat with no refined carbohydrate; and so had Stefansson (who subsisted when I knew him mainly on fatty meat and red wine) and McCarrison (who ate meat and whole-grain bread with quantities of butter but very little sugar), and neither was obese.

The ratio of EFA to certain non-EFA (mainly saturated and trans-polyunsaturated fatty acids) is the relevant consideration, rather than total fat or total saturated fat. Eskimos, when on their traditional diet, had the highest fat intake in the world and no dietary fibre, but even relatively long-lived ones had almost none of our non-infective "Western" diseases (ischaemic heart disease and other thrombotic disorders, cancer, diabetes mellitus, dental caries, multiple sclerosis, intestinal disorders, varicose veins). But they had a diet very high in EFA from fish and seal, and rapid intestinal transit times. Passmore leaves fish unchanged, and unfortunately McGovern states that the fat of fish should be reduced in the diet to help reduce total fat; whereas I would greatly increase it-especially our most caught fish, mackerel, which is readily available and cheap (about 35 p a pound), and contains more polyunsaturated than saturated fatty acids as well as some other nutrients, yet only a small pro- 